\title{
DISSOLUTION BEHAVIOR OF PHENYTOIN-BILE SALT COMPLEXES PREPARED BY CO-GRINDING
}

\section{Makoto OTSUKA* and Yoshihisa MATSUDA}

Kobe Pharmaceutical University, Motoyama-Kitamachi, Higashi-Nada, Kobe 658, Japan

The physicochemical properties of phenytoin (PHT)-bile salt complexes comprised of sodium dehydrocholate (DHCNa), sodium deoxycholate $(\mathrm{DCNa})$ or sodium cholate $(\mathrm{CNa})$ prepared by co-grinding were investigated by X-ray diffraction analysis, DSC and dissolution kinetics. All X-ray diffraction peak intensities of the co-ground PHT-bile salt [1 mol: $1 \mathrm{~mol}]$ mixtures were decreased by grinding for $3 \mathrm{~h}$, and showed a halo pattern of a noncrystalline solid. The solubility of ground products with DCNa, DHCNa and CNa were 212, 56, 68 times higher, respectively, than those of physical mixtures.

KEYWORDS dissolution behavior; mechanochemistry; phenytoin-bile salt complex; molecular interaction; solubility; noncrystalline solid

It is well known that components of bile such as bile salts, which are physiological surfactants, play an important role in intestinal absorption. ${ }^{1)}$ Many studies ${ }^{2,7)}$ have shown that bile salts markedly increase the solubility and dissolution rate of poorly water-soluble drugs, since the activity of bile salts is mainly due to micellar solubilization.

On the other hand, Miyata et al. ${ }^{8,9)}$ reported that bile acids form multimolecular inclusion compounds with a variety of organic substances in the solid state. Since the solid-state molecular interactions between the drugs and bile salts are affected by the enthalpy of the solid, interactions in the solidstate may affect the dissolution behavior of the drug and the bioavailability of pharmaceutical preparations. In this study, we prepared the phenytoin-bile salt complexes by a co-grinding method, and investigated their dissolution behavior.

\section{EXPERIMENTAL}

Grinding Conditions Equimolar mixtures of various kinds of bile salt, sodium dehydrocholate (DHCNa), sodium deoxycholate (DCNa) or sodium cholate $(\mathrm{CNa})$, and phenytoin $(\mathrm{PHT})$ were ground in a $350 \mathrm{ml}$ agate centrifugal ball mill at $360 \mathrm{rpm}$ for $3 \mathrm{~h}$.

Characterization Physicochemical properties of the ground complexes were characterized by X-ray powder diffraction analysis and differential scanning calorimetry (DSC).

Dissolution Behavior The dissolution profiles of the ground complexes were measured as follows: The sample powder containing $500 \mathrm{mg}$ PHT was introduced into $200 \mathrm{ml}$ of dissolution medium at $37 \pm 0.5^{\circ} \mathrm{C}$. The test solution was stirred by a paddle (JPXII) at $150 \mathrm{rpm}$. The drug concentrations of the samples contained in DCNa, DHCNa and CNa were determined spectrophotometrically at 224 and $280 \mathrm{~nm}$ to correct the absorption of the bile salts. Initial dissolution curves were determined as follows: A compressed pellet of the ground sample with $\mathrm{DCNa}$ fixed to the holder was set up on the dissolution instrument and rotated at $200 \pm 5 \mathrm{rpm}$ in $100 \mathrm{ml}$ of JPXII 2 nd fluid $(\mathrm{pH} 6.8)$ at $37 \pm 0.5^{\circ} \mathrm{C}$. 


\section{RESULTS AND DISCUSSION}

Effects of Co-grinding on Physicochemical Properties of PHT-Bile Salts System

The results of X-ray diffraction profiles of the co-ground PHT-bile salt [1 mol: $1 \mathrm{~mol}]$ mixtures were as follows: All diffraction peak intensities of the ground products were decreased by grinding and showed a halo pattern after $3 \mathrm{~h}$ grinding, suggesting that the mixtures transformed into a noncrystalline solid during grinding. Yakou et al. ${ }^{6}$ ) reported that the PHTDCNa coprecipitate containing more than $3 \mathrm{~mol}$ of DCNa was a stable noncrystalline solid, but the coprecipitate containing less than 2 mol of DCNa was not. They did not refer to the stoichometric PHT complex with bile acid. Therefore, their results suggested that the solid obtained by coprecipitation method was different from the equimolar complex obtained by grinding.

DSC curves of the ground products with PHT-DCNa, DHCNa or CNa showed 30,20 and $30^{\circ} \mathrm{C}$ lower melting points, due to that of the complex, respectively, than that of intact PHT, indicating the molecular interactions of these complexes were different from those of intact PHT crystals.

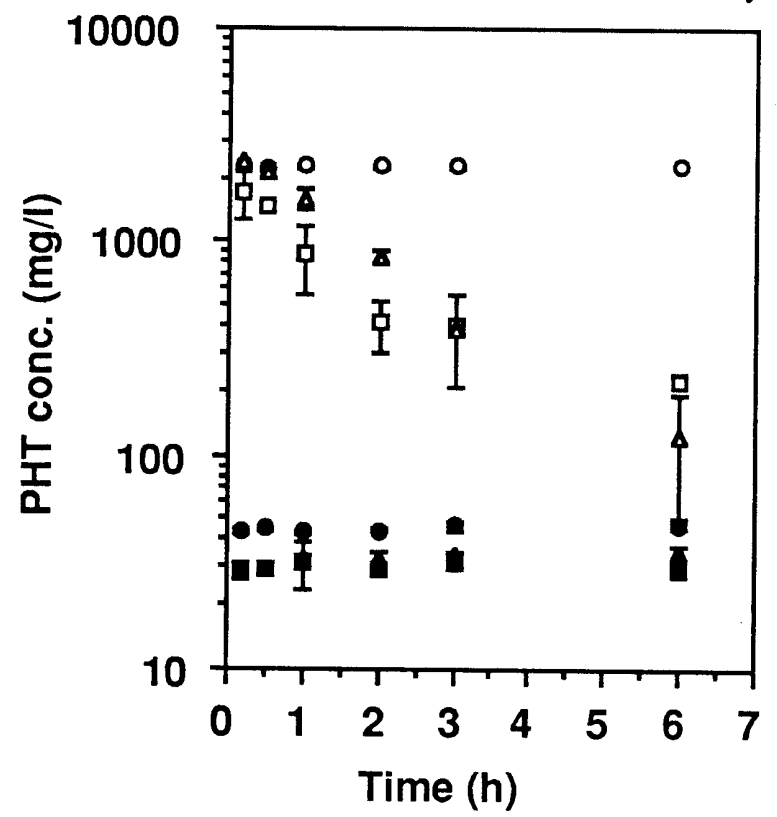

Fig. 1. Dissolution Behavior of Ground PHT-Bile Salt [1:1] Complexes at $\mathrm{pH} 6.8,37^{\circ} \mathrm{C}$

$(\mathrm{O}, \bullet)$, the products with $\mathrm{DCNa}$; $(\square, \boldsymbol{\square})$, with $\mathrm{DHCNa}(\Delta, \mathbf{\Lambda})$, with $\mathrm{CNa}$; open and closed symbols represent the ground products and physical mixtures, respectively. The bars represent standard deviation $(n=3)$.

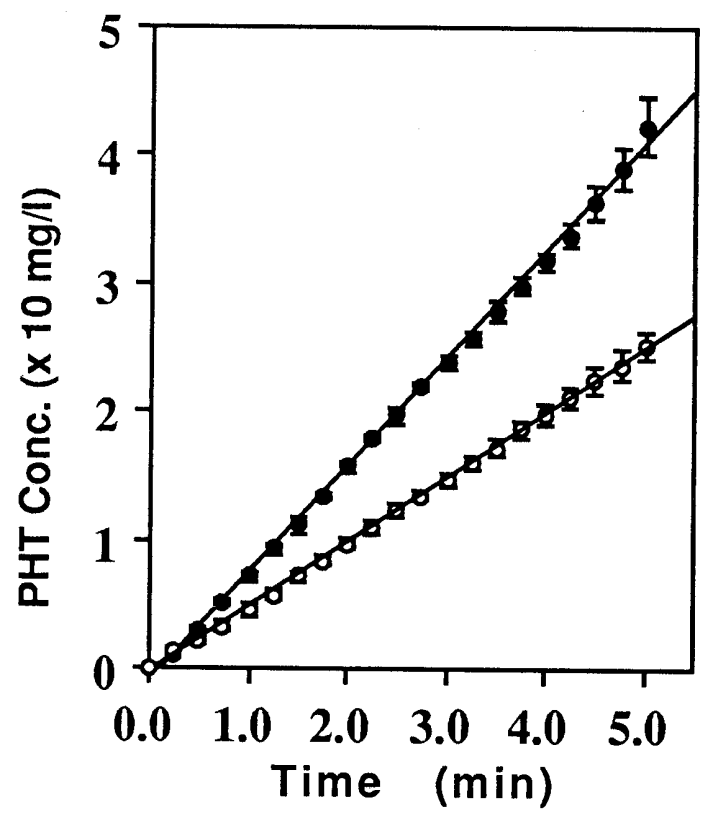

Fig. 2. Initial Dissolution Curves of Ground PHT-DCNa Complex at pH 6.8 and $37^{\circ} \mathrm{C}$ by the Rotating Disk Method

(๑), PHT-DCNa (1:1); (O ), PHT-DCNa (1:2); the bars represent standard deviation $(n=3)$.

\section{Dissolution Behavior of PHT-Bile Salt Complexes Obtained by Co-Grinding}

Figure 1 shows the dissolution profiles of $\mathrm{PHT}$ ground products with various kinds of bile salts at $\mathrm{pH} 6.8$; all PHT-bile salt ground products dissolved vary rapidly. The ground product with DCNa dissolved completely without any residue, and gave a constant drug concentration at $2460 \pm 11 \mathrm{mg} / 1$ during drug dissolution for $6 \mathrm{~h}$, indicating the solubility was higher than this constant value. On the other hand, the concentration of the ground products with $\mathrm{DHCNa}$ or CNa increased rapidly to give maximum concentrations of $1700 \pm 420$ and $2350 \pm 24.7 \mathrm{mg} / 1$, respectively, at $10 \mathrm{~min}$ and then declined to $223 \pm 14$ and $162 \pm 2.9 \mathrm{mg} / \mathrm{l}$ after $6 \mathrm{~h}$. The solubility of physical mixtures with DCNa, DHCNa and CNa were $47.9 \pm 1.8,29.7 \pm 1.0$ and $35.3 \pm 2.2 \mathrm{mg} / \mathrm{l}$, respectively. The maximum concentration of all ground products were much 
higher than those of the respective physical mixtures. Yakou et al. ${ }^{6}$ ) reported that the solubility of the PHT-DCNa complex (weight ratio, 1:5) obtained by coprepicitation in buffer at pH 6.8 was $120 \mathrm{mg} / \mathrm{l}$. Therefore, from these results it seems that the ground products were much more soluble than the solids obtained by co-precipition, indicating that molecular interactions in the ground products with bile salts were different from those obtained by the co-precipitation method. The ground complex with DHCNa or CNa dissolved rapidly at the initial dissolution stage; after that, the PHT concentration decreased with the elapse of time, since it was transformed into the stable form. However, the PHT concentration profile of the ground complex with DCNa showed a plateau. The dissolution behaviors suggested that the ground [1:1] complex with DHCNa or CNa was metastable in water, but that with DCNa was very stable.

\section{Evaluation of Solubility of PHT-Bile Salt Complexes by Initial Dissolution Kinetics}

In the previous section, the solubility of the ground PHT complexes [1:1] with DHCNa or CNa was estimated from Fig. 1, but the solubility of the ground PHT-DCNa complex was not determined, since all solid was dissolved in the solution. Therefore, to estimate the solubility of the ground PHT-DCNa complex, dissolution rate experiments were performed by the rotating disk method as reported by Sekiguchi and Kanke. ${ }^{10}$ )

$$
\mathrm{C}_{\mathrm{as}}=\mathrm{J}_{\mathrm{a}} \mathrm{C}_{\mathrm{hs}} / \mathrm{J}_{\mathrm{h}} \quad \text { eq. } 1
$$

where $\mathrm{C}_{\mathrm{as}}$ is the solubility of meta-stable form, $\mathrm{C}_{\mathrm{hs}}$ is the solubility of the stable form, $\mathrm{J}_{\mathrm{a}}$ is the initial dissolution rate constant of the metastable form, and $\mathrm{J}_{\mathrm{h}}$ is the initial dissolution rate constant of the stable form.

The solubility of the crystalline form of PHT was measured in $\mathrm{pH} 6.8$ buffer at $37^{\circ} \mathrm{C}$ by the powder dispersion method and estimated to be $27.2 \pm 0.17 \mathrm{mg} / \mathrm{l}(\mathrm{n}=5)$. Figure 2 shows the initial dissolution rate curves of ground PHT-DCNa [1:1] and [1:2] complexes in pH 6.8 buffer at $37^{\circ} \mathrm{C}$. The solubility of the ground PHT: DCNa [1:1] and [1:2] complexes were calculated from the initial dissolution rate constant using eq. 1 and estimated to be $10200 \pm 590$ and $6064 \pm 200 \mathrm{mg} / \mathrm{l}(\mathrm{n}=3)$, respectively. The solubility of the ground PHT-DCNa [1:2] complex was lower than that of the [1:1] complex. This result suggests that PHT:DCNa [1:1] complex formed the most watersoluble complex in the solid state, indicating that the finding was not due to micellar solubilization, but due to formation of [1:1] molecular complex. Therefore, we concluded that the solubility of PHT complexes with DCNa, DHCNa and CNa obtained by grinding were 212, 56 and 68 times higher than those of the physical mixtures, respectively. These results suggested that PHT formed water-soluble complexes with bile salts by mechanochemical treatment.

\section{REFERENCES}

1) K. W. Heaton, Bile Salts in Health and Disease, Churchill Livingston, The Whitefriars Press Ltd, London, (1972).

2) T. R. Bates, M. Gibaldi, J. L. Kanig, J. Pharm. Sci., 55, 191 (1966).

3) T. R. Bates, M. Gibaldi, J. L. Kanig, J. Pharm. Sci., 55, 901 (1966).

4) S. Miyazaki, H. Inoue, T. Yamahira, T. Nadai, Chem. Pham. Bull., 27, 2468 (1979).

5) S. Sakurai, S. Yakou, K. Fukumuro, K. Takayama, T. Nagai, M. Sugihara, Yakuzaigaku, 47, 260 (1987).

6) a) S. Yakou, S. Yamazaki, T. Sonobe, M. Sugihara, Yakuzaigaku, 46, 140 (1986);

b) S. Yakou, S. Yamazaki, T. Sonobe, T. Nagai, M. Sugihara, Chem. Pharm. Bull., 34, 3408 (1986).

7) S. Suzuki, Y. Miyazaki, S. Sakurai, S. Yakou, M. Sugihara, Yakuzaigaku, 50, 273 (1990).

8) M. Miyata, M. Shibakami, W. Goonewardena, K. Miki, Chemy. Lett., 605 (1987).

9) M. Miyata, M. Shibakami, S. Chirachanchai, K. Takemoto, N. Kasai, K. Miki, Nature, 343, 446 (1990).

10) M. Kanke, K. Sekiguchi, Chem. Pharm. Bull., 21, 878 (1973). 